Supporting Information

\title{
Excess Random Laser Action in Memories for Hybrid Optical/Electric Logic
}

Yu-Ting Cheng ${ }^{t}$, Chen-You Su $u^{*}, H$ sia-Yu Lin, Yu-Ming Liao, Tien-Lin Shen, Han-Wen $H u$, and Yang-Fang Chen*

Department of Physics, National Taiwan University, Taipei 106, Taiwan

*yfchen@phys.ntu.edu.tw 

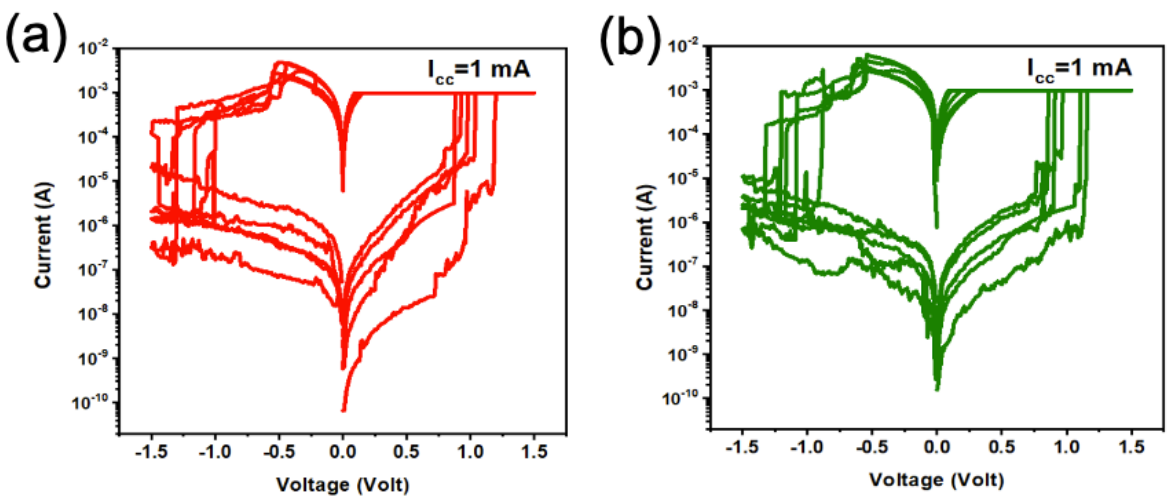

Figure S1. The I-V switching cycles of (a) the ITO/PMMA:QDs $\left(\boldsymbol{\lambda}_{\mathrm{em}}=630 \mathrm{~nm}\right) / \mathrm{Ag}$ CBRAM devices and (b) the ITO/PMMA:QDs $\left(\boldsymbol{\lambda}_{\mathrm{em}}=540 \mathrm{~nm}\right) / \mathrm{Ag}$ CBRAM devices. 
(a)

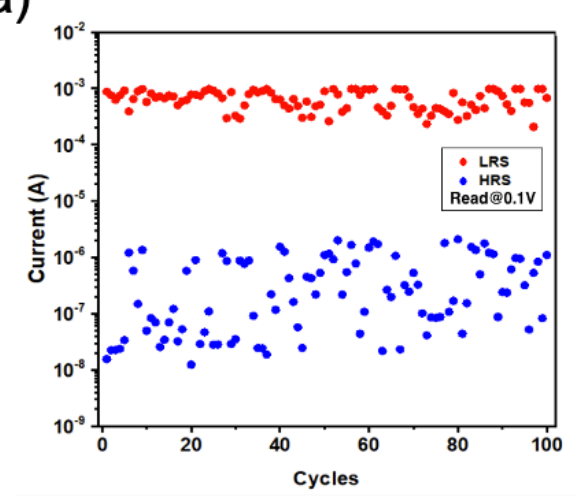

(b)

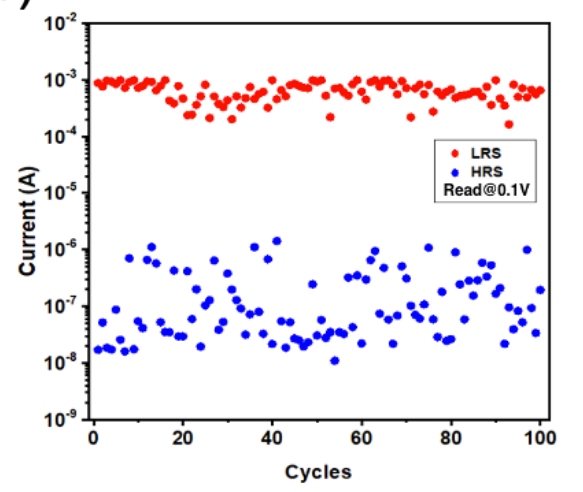

Figure S2. The DC endurance characteristic of (a) the ITO/PMMA:QDs $\left(\boldsymbol{\lambda}_{\mathrm{em}}=630\right.$ $\mathrm{nm}) / \mathrm{Ag}$ CBRAM devices and (b) the ITO/PMMA:QDs $\left(\boldsymbol{\lambda}_{\mathrm{em}}=540 \mathrm{~nm}\right) / \mathrm{Ag}$ CBRAM devices. 
(a)

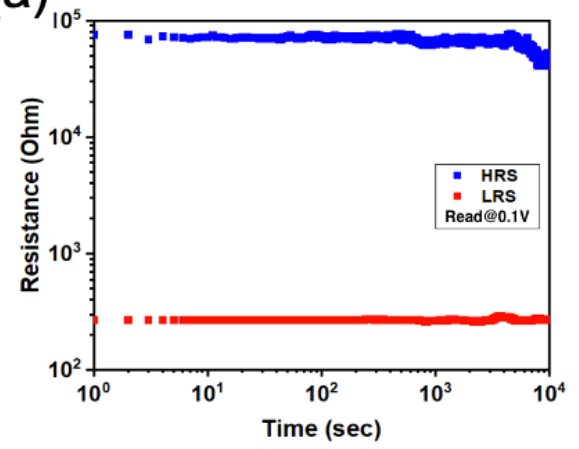

(b)

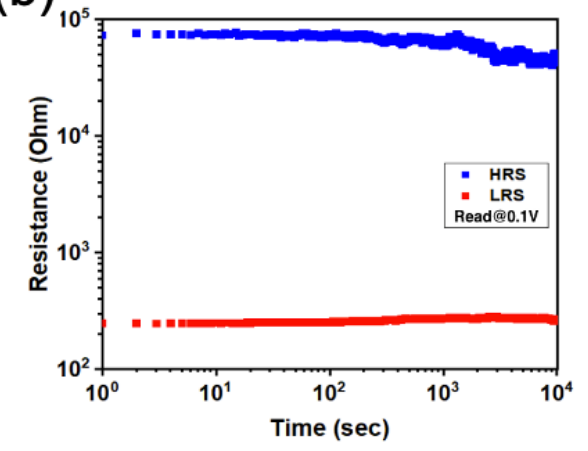

Figure S3. The resistance retention of (a) the ITO/PMMA:QDs $\left(\boldsymbol{\lambda}_{\mathrm{em}}=630 \mathrm{~nm}\right) / \mathrm{Ag}$

CBRAM devices and (b) the ITO/PMMA:QDs $\left(\boldsymbol{\lambda}_{\mathrm{em}}=540 \mathrm{~nm}\right) / \mathrm{Ag}$ CBRAM devices. 


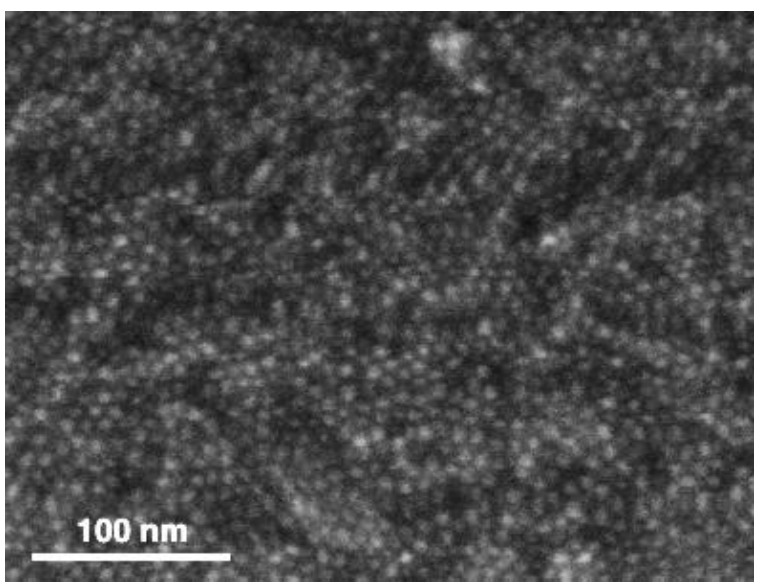

Figure S4. The SEM image of the QDs. 


\section{REFERENCES}

(1) Tsai, T.-L.; Chang, H.-Y.; Jiang, F.-S.; Tseng, T.-Y., Impact of Post-Oxide

Deposition Annealing on Resistive Switching in $\mathrm{HfO}_{2}$-Based Oxide RRAM and

Conductive-Bridge RAM Devices. IEEE Electron Device Letters 2015, 36, $1146-1148$.

(2) Suárez, I.; Gordillo, H.; Abargues, R.; Albert, S.; Martinez-Pastor, J., Photoluminescence waveguiding in $\mathrm{CdSe}$ and CdTe QDs-PMMA nanocomposite films. Nanotechnology 2011, 22, 435202. 